\title{
DIE WAHLEN VOM 28. NOVEMBER 1971 IN URUGUAY
}

\author{
Von ERNST-J. KeRbusch
}

\section{Das Ende des „Zweiparteiensystems“}

Uruguay, in den letzten Jahren von Unruhen erschüttert und durch spektakuläre Aktionen der Stadtguerilla der Tupamaros in die Schlagzeilen der Weltpresse gerückt, wählte am 28. November 1971 einen neuen Präsidenten und ein neues Parlament. Die Wahl war mit großer Spannung erwartet worden, da sich im letzten Jahr in dem kleinen Land zwischen den beiden mächtigen Nachbarn Argentinien und Brasilien eine Entwicklung angebahnt hatte, die Parallelen zu den Ereignissen des Vorjahres in Chile aufwies: in den beiden Ländern mit der längsten demokratischen Tradition auf dem südamerikanischen Subkontinent hatten sich Linksgruppierungen etabliert, die die Chance hatten, auf demokratischem Wege an die Macht zu gelangen. Was jedoch in Chile bereits eine lange Vorgeschichte hatte - schon von 1938 bis 1952 hatte eine Volksfront unter Führung der damals mittelständisch orientierten Radikalen das Land regiert ${ }^{1}$-, war für Uruguay ein Novum: Seit mehr als 100 Jahren hatte es nur zwei Parteien gegeben, die eine echte Chance besaßen, an die Macht zu gelangen: die städtisch orientierten - progressiveren Colorados und die mehr an den Interessen der Großgrundbesitzer orientierten - konservativeren - Blancos². Die beiden traditionellen Parteien hatten sich durch das Wahlsystem gegen Einbrüche dritter Gruppen abgesichert und die politische Szene des Landes bis 1966 unangefochten beherrscht. Sonstige Parteien kamen nie über eine unbedeutende parlamentarische Repräsentation hinaus. Echte politische Bedeutung besaßen sie nicht ${ }^{3}$.

Das schien sich bei der Wahl von 1971 erstmals geändert zu haben. Eine Linkskoalition unter Führung der Christdemokraten und der Kommunisten hatte sich gebildet, die nach Ansicht mancher Beobachter ${ }^{4}$ durchaus eine Chance besaß, die etablierten Parteien auszustechen: die Breite Front (Frente Amplio). Damit stellte sich zum ersten Male nicht mehr in erster Linie die Frage, welche der etablierten Parteien ein Mandat zur Regierungsbildung erhalten würde, sondern ob überhaupt ein weiteres Mal eine der beiden traditioneilen Parteien eine Mehrheit zu mobilisieren in der Lage sei.

\section{Der verfassungsrechtliche Hintergrund: Zwang zur Minderheitsregierungen}

Seit 1864 hatten Colorados und Blancos ihre Position im uruguayischen Parteiensystem unangefochten behauptet. Dabei waren die Colorados, die ihr wichtigstes

1 Für Einzelheiten zur politischen Entwicklung Chiles vgl. Urzúa Valenzuela, G., Los partidos políticos chilenos. Ensayos de insurgencia política en Chile, Santiago 1968, und Gil, F. G., The Political System of Chile, Boston 1966

2 Die Namen stammen noch aus der Zeit des großen Bürgerkrieges (1830-1834). Vgl. Pivel Devoto, J. E., Historia de los partidos políticos en el Uruguay, 2. Bd., Montevideo 1956, Bd. 1, S. 83. Die offiziellen Bezeichnungen lauten heute Partido Colorado und Partido Nacional.

3 Für Einzelheiten vgl. Kerbusch, E. J., Das uruguayische Regierungssystem. Der Zweite Colegiado 19521967, insbes. S. 99 ff

4 S. z. B. Peter, R., "Wird Uruguay ein zweites Chile?", in: Frankfurter Allgemeine Zeitung vom 27.11. 1971 und "Uruguay im Kielwasser Chiles. Bildung einer Volksfront“, in: Neue Zürcher Zeitung vom 12. 3. 1971. Auch eine Reihe von Umfrageergebnissen ließen diesen Schluß durchaus zu. S. z. B. die offizialistische Zeitung La Mañana noch einen Monat vor der Wahl. "La clase baja apoya al Partido Colorado y la clase más alta al Frente Amplio“, in: La Mañana vom 28. 10. 1971. 
Stimmenpotential vor allem aus den wachsenden städtischen Bevölkerungsschichten nahmen, fast hundert Jahre lang die "herrschende Partei“5 gewesen, deren Dominanz nur durch außergewöhnliche Umstände gebrochen werden konnte. Die chronische Schwäche der Blancos lag in ihrer Unfähigkeit begründet, städtische Wählerschichten langfristig an sich zu binden, da ihr eigentliches Reservoir, die Wähler auf dem Land, durch die Landflucht allmählich an Umfang verlor. Zum Ausgleich für die fehlende Chance des Machtwechsels hatten die Colorados den Blancos politische und personelle Zugeständnisse machen müssen, die zu einer Zusammenarbeit führte, die in Uruguay als "coparticipación" bezeichnet wurde6. Die coparticipación äußerte sich zunächst in politischen Verträgen, in denen die Verteilung von Interessensphären festgelegt wurde und später in gemeinsamen Vereinbarungen über Verfassungsreformen. Dabei erschien manchen uruguayischen Politikern ein System der kollegialen Exekutive, wie es in der Schweiz praktiziert wurde, als das geeignete System, um die coparticipación angemessen zu institutionalisieren. Bereits die Verfassung von 1918 sah eine kollegial organisierte Exekutive vor. So wurde das Kollegialorgan (Consejo Nacional de Administración) einem Präsidenten gleichberechtigt an die Seite gestellt. Die Verantwortung für die einzelnen Ressorts wurden auf Präsident und Consejo verteilt ${ }^{7}$. Diese Konstruktion ermöglichte es, einen Teil der Sitze im Consejo der Minorität zu überlassen, theoretisch ohne die Regierungsfähigkeit der Mehrheit zu gefährden ${ }^{8}$. Die Verfassung hatte allerdings nur so lange Bestand, wie schwache Präsidenten bereit waren, sich mit ihrer schwachen Rolle im Regierungssystem abzufinden und Konflikte zwischen den beiden Zweigen der Exekutive zu vermeiden. Nach einem Zwischenspiel von zwei Präsidialverfassungen (1934 und 1942), die jedoch ebenfalls Elemente der coparticipación enthielten, versuchte man es 1951 erneut mit einem Kollegialsystem - diesmal jedoch in unverfälschter Form: die Exekutive bestand nur noch aus dem 9 Mitglieder zählenden Consejo Nacional de Gobierno. Die stärkste Partei stellte 6, die zweitstärkste 3 Mitglieder. Diese Verfügung hätte - im Gegensatz zum eigentlichen Zweck der coparticipación - dritten Parteien zugute kommen können, wenn es ihnen gelungen wäre, Colorados oder Blancos als stärkste oder zweitstärkste Partei abzulösen. Der Bedrohung entzogen sich jedoch die beiden großen Parteien durch Bestimmungen im Parteien- und Wahlgesetz. Zuletzt ermöglichten sie die Akkumulierung von verschiedenen Gruppen unter einem gemeinsamen Namen (lema), schlossen jedoch alle Parteien ohne parlamentarische Vertretung von dieser Möglichkeit aus ${ }^{9}$. Dadurch wurde die Situation der kleineren Parteien, die ja kaum eine Chance hatten, die Stärke aller Gruppen einer der beiden traditionellen Parteien zusammen zu erreichen, sehr verschlechtert, da Stimmen für sie, zumindest was die Wahl der Exekutive anging, verloren waren, und der Eintritt neuer Gruppen in die Politik wurde außerordentlich erschwert. Andererseits wurde auch die Zersplitterung der beiden großen Parteien durch diese Gesetze gefördert, denn alle Untergruppen können eigene Kandidaten für die Präsidentenschaft und andere

5 Zum Begriff vgl. Duverger, M., Die politischen Parteien, Tübingen 1959, S. 317.

$6 \mathrm{~S}$. insbesondere Fonseca, M., La política de coparticipación, Montevideo 1951 und Pivel Devoto, J. E. Historia de los partidos políticos en el Uruguay, a.a.O., Bd. 1, S. $51 \mathrm{ff}$.

7 Eine ausführliche Darstellung und Diskussion findet sich bei Lindahl, G. G., Uruguay's New Path. A Study of Politics during the First Colegiado (1919-1933), Stockholm 1962; span. Ausgabe: Batlle, fundador de la democracia, Montevideo 1971.

8 Von den neun Mitgliedern des Consejo wurden alle zwei Jahre drei neu gewählt und im Verhälınis zwei zu eins auf die beiden stärksten Parteien verteilt. Da man mit einer Sechs-zu-drei-Verteilung der Colorados (die als Regierungspartei die Bedingungen weitgehend diktieren konnten) begonnen hatte, hätten die Blancos zwei aufeinanderfolgende Wahlen gewinnen müssen, um eine Mehrheit im Consejo zu erhalten. Dieser Fall trat aber während der Geltungsdauer der Verfassung von 1919 nie ein. S. Kerbusch, E. J., Das uruguayische Regierungssystem, a.a.O., S. 25.

9 Vgl. das Wahlgesetz von 1925 (Ley No. 7912 vom 22. 10. 1925) und die Ley de Lemas (Ley No. 9378 vom 5. 5. 1934). 
politische Ämter aufstellen. Dann werden die Stimmen aller Untergruppen eines lemas addiert und das stärkste lema zum Wahlsieger erklärt. Der Kandidat, der innerhalb dieses lemas die meisten Stimmen erhalten hat, ist gewählt. Der Integrationszwang, der von der Notwendigkeit ausgeht, einen gemeinsamen für alle akzeptablen Kandidaten zu finden, geht dadurch völlig verloren. Die unterlegenen Gruppen haben zudem auch für die parlamentarischen Ämter eigene Kandidaten aufgestellt - im Parlament werden die Sitze nach dem Verhältnis der Stimmen zwischen den einzelnen Gruppen vergeben ${ }^{\mathbf{1 0}}$-, die keineswegs bereit sind, den siegreichen Kandidaten zu unterstützen. Die Exekutive steht deswegen in aller Regel einem Parlament gegenüber, in dem gegnerische Mehrheiten den Ton angeben. $\mathrm{Da}$ aber das Regierungssystem nach der Verfassung auf einer Zusammenarbeit zwischen Exekutive und Legislative beruht - und das ist das gemeinsame Merkmal aller bisherigen Verfassungen Uruguays, gleich ob präsidentialistisch oder kollegialistisch -, liegt in diesem Gegensatz die Wurzel vieler Schwierigkeiten. Die Verfassungsänderung von 1966, von vielen Uruguayern nach der völligen Paralysierung des politischen Systems während des zweiten Colegiado sehnlich herbeigewünscht, brachte deswegen keine nennenswerten Veränderungen, weil sich an der zugrundeliegenden Struktur des Parteiensystems nichts änderte ${ }^{11}$. Der Präsident sieht sich seit 1969 den gleichen Schwierigkeiten gegenüber wie vorher der Consejo Nacional de Gobierno. Entscheidungen auf Mehrheitsbasis kommen heute wie vor 1967 kaum zustande. Allerdings ist der Präsident eher in der Lage, mit Hilfe des Ausnahmezustandes zu regieren als ein Rat von neun Mitgliedern, der mehr diskutiert als handelt. Der Präsident kann sich auch leichter über die Entscheidungen eines nichtkooperationswilligen Parlaments hinwegsetzen als ein Rat, dessen Mitglieder den verschiedenen parlamentarischen Gruppen selbst angehören. Das ist besonders wichtig bei einem Fall des lateinamerikanischen Präsidentialismus ${ }^{12}$, in dem der Institution des Präsidenten ohnehin mehr parlamentarische Kontrollen gegenüberstehen als z. B. dem Präsidenten der Vereinigten Staaten.

\section{Politische Parteien und Gruppierungen}

$\mathrm{Da}$ die lemas in Uruguay - wenn auch in der Regel als Parteien bezeichnet kaum als Parteien im eigentlichen Sinne, sondern eher als Wahlkoalitionen angesehen werden müssen ${ }^{13}$, hat sich das politische Bild in Uruguay seit den Wahlen von 1966 entscheidend gewandelt ${ }^{14}$. In den beiden großen Parteien haben sich entscheidende Umgruppierungen ergeben, und aus den politisch unbedeutenden, kleinen Parteien war eine dritte Kraft entstanden, die durch den Anschluß einiger prominenter Politiker aus den traditionellen Parteien noch an Schlagkraft gewonnen hatte. Von den drei großen politischen Gruppen - zu denen sich noch die Unión Radical Cristiana, eine bedeutungslose Abspaltung von den Christlichen

\footnotetext{
10 Das komplizierte Wahlverfahren wurde vom Verfasser an anderer Stelle eingehend erläutert. S. Kerbusch, E. J., Das uruguayische Regierungssystem, a.a.O., S. $47 \mathrm{ff}$.

11 Für eine ausführliche Diskussion der Verfassungsänderung und ihrer Auswirkungen s. Kerbusch, E. J., Uruguay in der Verfassungskrise, in: Jahrbuch Verfassung und Verfassungswirklichkeit, Band 4, No. 1-2, 1969, S. 195-218.

12 Zum Begriff vgl. Jiménez de Aréchaga, J., La constitución de 1952, 4 Bände, Montevideo 1952-1953, Bd. 1 , S. 11.

13 Darauf weisen u. a. bereits Taylor und Lindahl hin. S. Taylor, Ph. B. Jr., Interests and Institutional Dysfunction in Uruguay, in: The American Political Science Review, Bd. 51, No. 1, März 1963, S. 63 und Lindahl, G. G., Uruguay's New Path, a.a.O., S. $35 \mathrm{f}$.

14 Zur Parteiensituation und zur Wahl von 1966 vgl. Puhle, H. J., Politik in Uruguay, Vierteljahresberichte aus dem Forschungsinstitut der Friedrich-Ebert-Stiftung, Sonderheft 1, Hannover 1968, S. 32ff.
} 
Demokraten, hinzugesellte - hatte nur die Linkskoalition der Frente Amplio gemeinsame Kandidaten für die Ämter des Präsidenten, des Vizepräsidenten und der Intendenten der 19 Departamentos aufgestellt. In den anderen „Parteien“ bewarben sich jeweils mehrere Kandidaten.

\section{1) Die Colorados}

Am stärksten zersplittert zeigten sich die Colorados: Nicht weniger als fünf verschiedene Kandidaten präsentierțen sich dem Wähler, von denen allerdings nur zwei berechtigte Hoffnungen auf den Wahlsieg hatten. Eine der beiden aussichtsreichsten Gruppen der Colorados war die Unión Nacional Reeleccionista, die aus der 1966 von dem damals gewählten Präsidenten Gestido geführten Unión Colorada y Batllista und aus dem größeren Teil des Frente Colorado y Unidad hervorgegangen war. Die UNR betrieb die - nach der Verfassung unzulässige - Wiederwahl des amtierenden Präsidenten Pacheco und mußte deswegen versuchen, gleichzeitig mit dem Wahlgang ein Plebiszit über eine entsprechende Verfassungsänderung herbeizuführen. Für die Annahme der Verfassungsreform hätten mehr als fünfzig Prozent der Abstimmenden und mindestens fünfunddreißig Prozent der Abstimmungsberechtigten stimmen müssen. Da wegen der Wahlpflicht mit einer hohen Wahlbeteiligung zu rechnen war, hätten etwa achthunderttausend Stimmen für das Plebiszit abgegeben werden müssen - eine Unmöglichkeit angesichts der Ablehnung der Reform durch die anderen Gruppen der Colorados und die anderen lemas. Für den Fall einer Ablehnung des Plebiszites hatte Pacheco seinen Landwirtschaftminister Juan Maria Bordaberry zum Kandidaten nominiert ${ }^{15}$. Bordaberry hatte 1959 dem Parlament als Senator der Blancos angehört. Deshalb sprengte der „einsame Entschluß“ des Präsidenten die UNR, und einige prominente Mitglieder traten aus der UNR aus. Diese Spaltung schien zunächst die Chancen der traditionsreichsten Coloradogruppe, der von Jorge Batlle angeführten Liste „15“ (Unidad y Reforma) $\mathrm{zu}$ erhöhen. Aber Jorge Batlle, ein hochintelligenter und schlagfertiger Jurist, fehlte das Charisma seines Großonkels José Batlle (Präsident von 1903 bis 1907 und von 1911 bis 1915) und seines Vaters Luis Batlle (Präsident von 1947 bis 1950 und 1955 Präsident des Consejo Nacional de Gobierno). Neben Pacheco bzw. Bordaberry und Jorge Batlle gab es noch eine „dritte Front" der Colorados, in der zwei gemäßigt linksgerichtete Senatoren, Amílcar Vasconcellos und Manuel Flores Mora, sich zusammengeschlossen hatten. Die Koalition der beiden in kompromißloser Opposition zur Pachecoregierung stehenden Politiker vergrößerte paradoxerweise - wegen der Kumulierung der Stimmen - die Chancen der Reeleccionistas, da die Stimmen der "dritten Front" letztlich der stärksten Untergruppe der Colorados zugute kamen ${ }^{16}$.

\section{2) Die Blancos}

Den Blancos war es im Laufe des Jahres 1971 nach langjährigen Verhandlungen und mehreren gescheiterten Versuchen gelungen, wieder ein einheitliches Directorio - das heißt eine Parteiführung, die von allen Gruppen akzeptiert wurde - zu

15 Im Falle der Wiederwahl Pachecos war Bordaberry als Kandidat für die Vizepräsidentschaft nominiert.

16 Dieses paradoxe Resultat stellte sich auch prompt ein: ohne die ca. 45000 Stimmen des Tercer Frente hätte der Partido Nacional die Wahl sicher gewonnen: S. für die Zahlen (vorläufiges offizielles Endergebnis des Innenministeriums) "Resultados finales del escrutinio primario“, in: El Popular vom 4.12 .1971$. 
wählen ${ }^{17}$. Die Hoffnungen, daß es nach dieser Einigung möglich sein würde, auch einen gemeinsamen Präsidentschaftskandidaten zu wählen, erfüllten sich jedoch nicht. Die konservativen Gruppen, die 1966 die Alianza Nacionalista gebildet hatten, und von dem alternden Caudillo Martín Echegoyen (Herrerismo) und von Alberto Heber (Heberismo) angeführt wurden, lehnten jeden auch noch so vorsichtig progressiv wirkenden Kandidaten ab, und die fortschrittlichen Gruppen standen einer Kandidatur des angesehenen, aber stark rechts ausgerichteten Alberto Gallinal Heber, einem der mächtigsten Großgrundbesitzer Uruguays, der als einziger für eine gemeinsame Kandidatur infrage gekommen wäre, sehr skeptisch gegenüber. Vor allem der von Gallinal selbst begründete Movimiento de Rocha lehnte seinen früheren Führer ab. Gallinal stellte sich deswegen nicht für eine Kandidatur zur Verfügung und machte den Weg frei für die Kandidaturen des Exgenerals Mario Aguerrondo - mit Alberto Heber als Vizepräsidentschaftskandidat - und des Senators und früheren Landwirtschaftsministers Wilson Ferreira Aldunate, dessen neugebildete Gruppe "Por la Patria“ sich aus den Restbeständen einiger aufgelöster Blancogruppierungen zusammensetzte. Der neue Führer des Movimiento de Rocha, Carlos Julio Pereyra, kandidierte neben Ferreira für die Vizepräsidentschaft. Die Chancen der Präsidentschaftsformel Ferreira/Pereyra waren von Anfang an besser als die des unflexiblen Aguerrondo, dessen einziger Wahlslogan, das Versprechen der Wiederherstellung von Ruhe und Ordnung, kaum ankam, da man das eher von den Colorado-Kandidaten - insbesondere von Pacheco selbst - erwartete. Da aber die Blancos nur zwei Kandidaten aufgestellt hatten, war vorauszusehen, daß der stärkste Kandidat der Blancos den stärksten Kandidaten der Colorados deutlich überflügeln würde. Erneut war also eine Präsidentschaft zu erwarten, die sich nur auf eine winzige Minderheit im Parlament stützen konnte.

\section{3) Der Frente Amplio}

Aus den - schon wegen des Wahlsystems - politisch bedeutungslosen kleineren Parteien entstand Ende 1970/Anfang 1971 eine neue politische Gruppierung, die das traditionelle "Zweiparteiensystem" 18 Uruguays infrage stellte. Kommunisten, - seit 1962 bereits im Frente Izquierda de Liberación (FIdeL) mit einigen anderen Linksgruppen zusammengeschlossen -, Christdemokraten, Sozialisten und eine Gruppe von Unabhängigen schlossen sich zum Frente Amplio, einer „breiten Front“ zusammen ${ }^{19}$, der sich später der Movimiento Blanco Popular y Progresista unter dem Blanco-Senator Rodríguez Camusso und die Colorado-Senatoren Michelini und Alba Roballo anschlossen. Die neugebildete Gruppierung konnte sich zwar nach komplizierten Verhandlungen auf gemeinsame Kandidaten für die Ämter von Präsident, Vizepräsident und Intendenten der 19 Departamentos einigen, gemeinsame Listen für Senat und Abgeordnetenhaus ließ die Heterogenität der einzelnen

\footnotetext{
17 S. „El domingo asume el nuevo directorio de los Blancos“, in: Carta de Montevideo, Bd. 6, No. 298, vom 22. 3. 1971, S. 2.

18 Zur Problematik dieser Kennzeichnung wurde oben bereits Stellung genommen. S. auch Kerbusch, E. J., Das uruguayische Regierungssystem, a.a.O., S. 109f.

19 Für eine Dokumentation vgl. „Frente Amplio Cristianos y Marxistas“, „Frente Amplio“ und „Frente Amplio. Respuesta al despotismo“, Cuadernos de Marcha, Nos. 46 (Februar 1971), 47 (März 1971) und 53 (September 1971). Außerdem Mini informe No. 4 ¿Qué es el 26 M? und Mini informe. No. 6 ¿Qué es el Frente Amplio? beide Montevideo o. J. (1971). Für Darstellungen der Führer der beiden bedeutendsten Gruppen des Frente Amplio vgl. Terra, J. P. (Partido Demócrata Cristiano), Del Pachequismo al Frente Amplio, Montevideo 1971 und Arismendi, R. (Frente Izquierda de Liberación), La revolución uruguaya en la hora del Frente Amplio, Montevideo 1971.
} 
Gruppen jedoch nicht zu. Um trotz eigener Listen ihre Stimmen für Präsident und Intendenten kumulieren zu können, mußte sich der Frente Amplio eines lemas bedienen, das bereits 1966 parlamentarische Repräsentation errungen hatte. Da der Name FIdeL den Frente Amplio zu sehr mit den Kommunisten indentifiziert hätte, einigte man sich auf das lema Partido Demócrata Cristiano - wohl der bisher einzige Fall in der Geschichte, daß auch eine kommunistische Partei unter dem Namen Christliche Demokraten kandidierte.

Der Frente Amplio sah seine Chance vor allem in der Hauptstadt Montevideo, auf die sich auch die Hauptaktivitäten konzentrierten. Als Einheitskandidat wählte man den angesehenen parteilosen und als fortschrittlich bekannten Exgeneral Líber Seregni und stellte ihm die ebenfalls parteilosen Mediziner Crottogini (Vizepräsidentschaft) und Hugo Villar (Intendenz von Montevideo) zur Seite. Die Kandidaten des Frente Amplio wurden bereits am 26. März proklamiert ${ }^{20}$ - lange bevor die anderen Parteien ihre Kandidaten benannt und den Wahlkampf eröffnet hatten.

\section{Die innenpolitische Situation im Wahlkampfjahr und der Wahlkampf}

Der vom Frente Amplio früh begonnene Wahlkampf zersplitterte das ohnehin aktionsunfähige Parlament weiter, da drei Senatoren aus den traditionellen Parteien und mehrere Abgeordnete dem Frente Amplio beigetreten waren. Die Konflikte zwischen Exekutive und Legislative nahm immer härtere Formen an. So lehnte die Comisión Permanente bereits im März die von der Exekutive beantragte Verschärfung des Ausnahmezustandes durch die Aufhebung der individuellen Freiheitsrechte ${ }^{21} \mathrm{ab}$ - ohne jedoch die Exekutive daran zu hindern, politische Gefangene trotz richterlicher Freilassungsordnung in Gefangenschaft $z u$ halten und in speziell dafür eingerichteten Konzentrationslagern zusammenzufassen. Die Schließung von oppositionellen Zeitungen durch die Exekutive wurde vom Parlament scharf mißbilligt und führte zu einer allerdings erfolglosen Interpellation des Innenministers Brum Carbajal. Auch zwischen Exekutive und dem Obersten Gerichtshof kam es zu Konflikten: das Oberste Gericht - machtlos gegen die Praxis der Internierung politischer Gefangener ohne richterlichen Beschluß - wehrte sich gegen angebliche Eingriffe des Innenministers in die Unabhängigkeit der Justiz ${ }^{22}$.

Die Konflikte zwischen den Staatsgewalten gipfelten schließlich in der von den Blancos beantragten Aufhebung des Ausnahmezustandes durch die Asamblea General am 14.7.1971 und dessen sofortiger Wiederverhängung durch die Exekutive, die zur Beantragung eines politischen Prozesses wegen Verfassungsbruches gegen den Präsidenten führte ${ }^{23}$. Die weitere Behandlung des „juicio político“, der wie alle früheren Versuche, auf dem Wege einer Anklage einen Präsidenten zu stürzen, im Parlament „einschlief“, zeigte eindeutig die echten Machtverhältnisse in Uruguay: der Präsident hatte den Konflikt mit Legislative und Judikative durch Dominanz gelöst, und die beiden unterlegenen Gewalten machten nur noch schüch-

$20 \mathrm{Vgl}$. „Proclamación del General Líber Seregni“, in: Cuadernos de Marcha Nr. 46 (Februar 1971), S. 33ff.

21 Vgl. "La Comisión Permanente no autorizó la suspensión de las garantías individuales“, in: Carta de Montevideo, Bd. 7, No. 296 vom 8. 3. 1971, S. 3.

22 Innenminister Danilo Sena wurde beschuldigt, von dem zuständigen Richter die sofortige Freilassung eines festgenommenen Kriminalbeamten gefordert zu haben. Auch in diesem Falle scheint die Anschuldigung der Suprema Corte eher gegen Pacheco selbst als gegen seinen Minister gerichtet gewesen zu sein. S. "Conflicto de poderes: Suprema Corte vs. P. E.“, in: Carta de Montevideo, Bd. 7, No. 310, vom 14. 7. 1971, S. 3.

23 S. "Tupamaros als Gesetzgeber“, in: Frankfurter Allgemeine Zeitung vom 26. 8. 1971. 
terne Versuche, mit gelegentlichen Anklagen und Querschüssen gegen diesen „Mißbrauch “ 24 zu protestieren.

Die Aufrechterhaltung des Ausnahmezustandes - auch über die Wahl hinaus gab der Regierung die Möglichkeit, eine allzu extensive Wahlkampagne des Frente Amplio zu verhindern. Trotzdem lag Pacheco an der Aufrechterhaltung der Fiktion des völlig freien Wettbewerbs zwischen den politischen Gruppierungen. So beschränkten sich die Gegenmaßnahmen gegen den früh eröffneten Wahlkampf des Frente Amplio ${ }^{25}$ auf die regelmäßige Schließung von dessen Zeitungen mit oft fadenscheinigen Begründungen.

Von August an nahm bei Gallupumfragen der Partido Colorado die Spitzenposition ein. Auch die Wahlintention für die Blancos nahm zu, während sich die Zahl der Unentschlossenen mit dem Näherrücken des Wahltages verminderte und der Stamm der Sympathisanten des Frente Amplio ziemlich konstant blieb.

Hinzu kam der Vorsprung der traditionellen Parteien in den ländlichen Departamentos des Inneren, in denen der Frente Amplio kaum Fuß gefaßt hatte.

Die Wahlkampfführung aller Parteien unterschied sich 1971 grundsätzlich von der Praxis früherer Wahlkämpfe. Die Entstehung einer den großen Parteien fast gleichwertigen dritten Kraft forderte völlig neue Strategien. Hatte sich bei früheren Wahlen der Wahlkampf der traditionellen Parteien fast ausschließlich nach Innen (also gegen sublemas der gleichen Partei, bei denen man eher Stimmen zu holen hoffte als bei der gegnerischen Partei) gerichtet, so hatten die beiden "demokratischen" Parteien sich diesmal gemeinsam gegen einen "totalitären“ Gegner zu wenden. In einer intensiven Wahlkampagne in allen Blanco- und Coloradozeitungen wurde schon Monate vor der Wahl in ganzseitigen Anzeigen vor dem Kommunismus, der nach Chile nun auch Uruguay zu erobern suche ${ }^{26}$, gewarnt.

Zwar hatten alle politischen Gruppen Regierungsprogramme ausgearbeitet ${ }^{27}$, aber die traditionelle Bedeutungslosigkeit solcher Papiere ließ auch diesmal kaum nennenswerte Aufschlüsse über die tatsächlichen Absichten der chancenreichsten Gruppen zu. Außerdem stand bereits vor der Wahl fest, daß der siegreiche Kandidat über keine Mehrheit im Parlament verfügen würde. Er mußte also in jedem Fall mit anderen Gruppen zu einem politischen Kompromiß kommen, um wenigstens einen Teil seiner Pläne verwirklichen zu können. Die Colorados (insbesondere die Reelecionistas) hatten kaum mehr als eine Fortsetzung der bisherigen Politik der Stärke anzubieten, die - wie die Meinungsumfragen bewiesen - besonders bei den unteren Sozialschichten zunehmend populär wurde. Wilson Ferreira Aldunate versprach vor allem eine Agrarreform sowie die Nationalisierung des Bankensektors und des Außenhandels. An dieses gemäßigte reformerische Programm glaubte man

24 Eigentlich handelt es sich um einen in Krisenzeiten durchaus "natürlichen “ Vorgang. Das ungleichgewichtige uruguayische Regierungssystem beruht theoretisch auf einem "Gleichgewicht ${ }^{\text {" }}$ zwischen den Staatsgewalten (checks and balances). Wenn dann in Krisenzeiten unterschiedliche politische Kräftekonstellationen in den einzelnen Staatsgewalten die Handlungsfähigkeit der Regierung blockieren, wird ein starker Präsident den Versuch unternehmen, das so entstehende Patt durch Dominanz zu durchbrechen. Zum Fall der USA, die dieses Problem ebenfalls kennen, vgl. Hermens, F. A., Verfassungslehre, Frankfurt 1964, S. 532f. Für Uruguay vgl. Kerbusch, E. J., Das uruguayische Regierungssystem, a.a.O., S. 128.

25 Dem Frente Ampa erschien diese frühe Eröffnung des Wahlkampfes nötig, da er als neue Kraft in der Bevölkerung erst bekannt werden mußte. Das machte sich allerdings in der Endphase des Wahlkampfes nach Beobachtungen des Verfassers mit der fast vollständigen physischen Erschöpfung der Spitzenkandidaten bemerkbar.

26 Die "totalitären“ Methoden wandten allerdings die „demokratischen“ Kräfte an, die sich weder an das Verbot von Wahlkampfveranstaltungen am Vorwahltag hielten noch die Bannmeile um die Wahllokale respektierten und die am letzten Tag vor der Wahl unter Polizeischutz die Plakate des politischen Gegners mit eigenen überklebten (So die U. N. R. „Reeleccionismo“). S. auch die Zeitungsanzeigenserien wie z. B. "Alerta Uruguay“, "Encuesta “ und "Derrotales“ in La Mañana, El País, El Diario, Acción, La Democra"Aia etc., deren Auftraggeber ungenannt blieb. Für eine Stellungnahme des Frente Amplio vgl. "Los Yanquis prosigen su campana de terror “, in: El Eco vom 6. 12. 1971.

27 Partido Nacional, Declaración de principios und Nuestro compromiso con Usted, beide Montevideo 1971 und UNR, La U. N. R. presenta al pueblo su plan de gobierno, Montevideo, Oktober 1971. 
allerdings nur mit Einschränkungen. Ferreiras erfolgloser Versuch einer Agrarreform als Landwirtschaftsminister der Blancoregierung von 1963 bis 1967, der vor allem an Widerständen in der eigenen Partei gescheitert war ${ }^{28}$, mahnte zur Skepsis, denn auch eine Regierung unter Ferreiras Führung war nur in einer Koalition mit anderen Gruppen möglich; und deren Zustimmung zu einer Agrarreform war zumindest fraglich.

Der Frente Amplio hatte zwar als einzige Partei mit seinen „dreißig ersten Maßnahmen der Regierung" ein Sofortprogramm vorgelegt, das aber auch nur als Diskussionsgrundlage hätte nützlich sein können, da auch für den Frente Amplio im Falle eines kaum denkbaren Wahlsieges das Problem der Mehrheitsfindung im Parlament entstanden wäre29. Das Programm des Frente Amplio glich äußerlich in vielen Einzelpunkten dem Wahlprogramm Ferreira Aldonates und kündigte in seinem ersten Abschnitt vier fundamentale Maßnahmen an:

a) eine Agrarreform,

b) die Nationalisierung des Bankensektors,

c) die Nationalisierung der wichtigsten Sparten des Außenhandels und

d) eine energische industrielle Aktivität des Staates, die die Nationalisierung der Schlachthöfe einschließt.

Außerdem versprach der Frente Amplio die Wiederherstellung der rechtsstaatlichen Ordnung, eine Neuverteilung der Einkommen und die Befriedung des Landes. Während das Programm Ferreiras jedoch nicht überall ernst genommen wurde, erwartete man vom Frente Amplio, daß er versuchen würde, sein Programm im Falle eines Wahlsieges auch in die Tat umzusetzen. Das Beispiel der Unidad Popular in Chile dürfte zu dieser weitverbreiteten Überzeugung erheblich beigetragen haben ${ }^{30}$.

Eine wichtige Rolle im Wahlkampf spielten auch die Tupamaros, die für den Wahlkampf einen Waffenstillstand angekündigt hatten, den sie auch einhielten. Aber sie hielten immer noch vier Politiker und Industrielle - teilweise schon seit vielen Monaten - gefangen. Die offizialistischen Zeitungen versuchten, den Frente Amplio als den politischen Zweig der Tupamaros darzustellen ${ }^{31}$, dem alle demokratischen Eigenschaften abzusprechen seien. Auf der anderen Seite klagte die Linke die Regierung an, den einigen hundert politisch Gefangenen gegen den Spruch des obersten Wahlgerichtes das Stimmrecht zu verweigern ${ }^{32}$. Darin stimmten ihr sogar die konservativen Gruppen der Blancos, die Aguerrondo unterstützten, $\mathrm{zu}^{33}$. Nachrichten über eine "goodwill“-Aktion der Tupamaros, die Freilassung des fast fünf Monate vorher entführten Industriellensohnes Jorge Berenbau zwei Tage vor der Wahl, durften auf Anweisung der Regierung nicht veröffentlicht werden. Die Jorge Batlle unterstützende Zeitung El Día wurde für zwei Tage verboten, da sie sich unter Berufung auf Pressefreiheit und Informationspflicht nicht an diese Anordnung hielt ${ }^{34}$.

28 Vgl. Kerbusch, E. J., Das uruguayische Regierungssystem, a.a.O., S. 125.

29,30 primeras medidas de gobierno“, abgedruckt in: Frente Amplio. Respuesta al despotismo, Cuadernos de Marcla No. 53, September 1971, S. 75-80.

30 Nohlen weist zwar mit Recht darauf hin, daß Unidad Popular in Chile und Frente Amplio in Uruguay nicht ohne Einschränkungen vergleichbar sind. Die Ausstrahlungskraft des Wahlsieges Allendes auf die Situation in Uruguay darf aber auch nicht unterschätzt werden. S. Nohlen, D., Politischer Wandel durch Wahlen: Der Fall Uruguay, a.a.O., S. 14.

31 Vgl. z. B. "Conexión de sediciosos con el F. A.“, in: La Democracia vom 24. 11. 1971.

32 S. "Los 200 que no votarán mañana“, in: El Eco vom 27. 11. 1971.

33 S. "Respetar a la Corte Electoral“, in: La Democracia vom 22.11. 1971.

34 S. „Berembau libre, según afirman tres testigos“, in: El Día vom 27. 11. 1971. 


\section{Wahlergebnis und Probleme der Regierungsbildung}

Auf das Ergebnis der Wahl vom 28. November 1971 mußten die Uruguayer länger warten als je zuvor: Erst am 3. Dezember konnte das Innenministerium ein vorläufiges offizielles Endergebnis vorlegen, nach dem die Colorados mit dem knappen Vorsprung von 9596 Stimmen in Front lagen - ein denkbar knapper Vorsprung in Anbetracht der Tatsache, daß fast 200000 Stimmen in der Auszählung noch nicht enthalten waren: die votos observados ${ }^{35}$ und die votos interdepartamentales ${ }^{36}$.

Die Informationspolitik der Regierung löste aufgrund vieler Unstimmigkeiten bei den Blancos und dem Frente Amplio den verständlichen Verdacht der Manipulation aus, zumal Urnen nicht auffindbar waren und teilweise mehr Stimmen als Wahlberechtigte vorhanden zu sein schienen ${ }^{37}$.

Obwohl mit einem endgültigen Wahlergebnis erst für Februar zu rechnen war, waren bereits wenige Tage nach der Wahl die wichtigsten Tendenzen erkennbar: Pachecos Wiederwahlabsicht war fehlgeschlagen; das Plebiszit hatte nur die Unterstützung von etwa $28 \%$ Wähler erhalten ${ }^{38}$. Der Frente Amplio hatte trotz eines im Vergleich zur Wahl von 1966 achtbaren Resultates ${ }^{39}$ die hochgespannten Erwartungen in keiner Weise erfüllen können. Auch in Montevideo konnte er die Colorados nicht gefährden, die über einen sicheren Vorsprung von mehr als 50000 Stimmen verfügten. Sogar die Blancos blieben in Montevideo nur mit etwa 5000 Stimmen hinter dem Frente Amplio zurück ${ }^{40}$. Auf der anderen Seite erzielte in Montevideo der Kandidat des Frente Amplio die meisten Stimmen, da sich die Stimmen der Colorados und Blancos jeweils auf mehrere Kandidaten verteilten. Durch die Ley de Lemas wurde diese Mehrheit jedoch nicht wirksam: dem Kandidaten der Unión Nacional Reeleccionista genügten 150000 Stimmen, um Hugo Villar trotz dessen 200000 Stimmen den Weg zur Intendenz zu versperren, denn die anderen Colorado-Gruppen steuerten weitere 100000 Stimmen bei.

Ähnlich sah es zwischen Bordaberry, dem stärksten Coloradokandidaten, und Wilson Ferreira Aldonate, dem stärksten Blanco-Kandidaten, aus: zwar erreichte Ferreira mit 440000 Stimmen die bei weitem größte Stimmzahl, aber zu Bordaberrys 379000 Stimmen addierten sich noch die seiner innerparteilichen Gegner, und die erwiesen sich mit insgesamt 299000 Stimmen stärker als Mario Aguerrondo, der es trotz der traditionellen Stärke des Herrerismo in den meisten ländlichen Departamentos nur auf 229000 Stimmen brachte.

Trotz erheblicher Überlegenheit im Inland gelang es den Blancos nicht, an die Colorados heranzukommen, denn deren Vorsprung in Montevideo, das allein über mehr als $40 \%$ der Wähler verfügt, war zu groß. Die endgültige Auszählung der Stimmen, die von den Juntas Electorales vorgenommen wurde, mußte allerdings den knappen Vorsprung des Partido Colorado zuerst noch bestätigen. Das Aus-

35 Wähler, die sich nicht lückenlos ausweisen können oder deren Eintragung in das Wahlregister noch nicht bestätigt ist etc. wählen ,observados“ $-\mathrm{d}$. h. unter Beobachtung - und weisen sich durch ihren Fingerabdruck aus. Ưber die Gültigkeit der Stimme entscheidet bei der endgültigen Auszählung die Junta Electoral.

36 Stimmen, die in Departamentos abgegeben werden, in denen der Wähler nicht registriert ist. Sie werden später dem entsprechenden Depatamento zugeschlagen. Die meisten votos interdepartamentales werden in Montevideo für Departamentos im Inneren des Landes abgegeben.

37 S. Chifflet, G., La elección y sus sombras, in Marcha No. 1578 vom 14. 1. 1972, S. 9.

38 Die Zahlen wurden vom Verfasser zusammengestellt aus "Resultado oficial del escrutinio primario“, in: Ahora vom 3. 12. 1971 und "Escrutinio primario general “, in: El Día vom 4. 12. 1971.

39 Der Frente Amplio hatte seiren Stimmenanteil von 13,1\% auf 18,7 \% steigern können. Diese Zahl erscheint bei der Schwierigkeit des Vergleichs - die einzelnen Gruppen hatten ja 1966 in anderen Koalitionen kandidiert, deren Stimmen nicht mit Sicherheit zugeordnet werden können - einigermaßen realistisch. Sie ist entnommen aus "La moraleja del Uruguay“, in: Analisis (Buenos Aires), Bd. 11, No. 559 vom 3. 12. 1971, S. 9.

40 S. "Escrutionio primario general“, in: El Día vom 4. 12. 1971. 
zählverfahren - durch ständige Einsprüche und Vorwürfe des Partido Nacional und des Frente Amplio wegen tatsächlicher oder vermeintlicher Unregelmäßigkeiten wiederholt unterbrochen - ging schließlich so langsam vor sich, daß die Befürchtung aufkam, bis zur Neuwahl der neuen Präsidenten des Abgeordnetenhauses und des Senates (am 15. 2. 1972) werde noch gar kein endgültiges Ergebnis vorliegen.

Am 11. Februar - vier Tage vor dem Amtsantritt der neuen Kammern - konnte schließlich das endgültige offizielle Ergebnis der Wahl vom 28. November 1971 bekanntgegeben werden: es bestätigte den wahlsieg der Unión Nacional Reeleccionista. Der Vorsprung der Colorados hatte sich sogar auf etwas mehr als 12000 Stimmen, das sind 0,7\% der angegebenen Stimmen, vergrößert. Der neue Präsident José María Bordaberry, jetzt seines knappen Wahlsieges sicher, konnte nun erst versuchen, sich eine parlamentarische Basis für die zukünftige Regierungsarbeit zu suchen.

Tabelle I

Ergebnis der Wahl vom 28. November 197141:

\begin{tabular}{llrr} 
Partei & Kandidat & Stimmen & in \% \\
\hline Partido Colorado & Bordaberry & 379157 & 22,9 \\
& Battle & 243320 & 14,7 \\
& Vasconcellos & 56001 & 3,4 \\
\cline { 2 - 4 } Partido Colorado & insgesamt: & 681624 & 41,0 \\
Partido Nacional & Ferreira & 439649 & 26,5 \\
& Aguerrondo & 228558 & 13,8 \\
Partido Nacional & insgesamt: & 668822 & 40,3 \\
PDC (Frente Amplio) & Seregni & 304275 & 18,3 \\
Sonstige & & & 0,4 \\
\hline Stimmen insgesamt: & & 1664119 & $100 \%$
\end{tabular}

Tabelle II

Die Verteilung der Mandate in Senat und Abgeordnetenhaus ${ }^{42}$ :

\begin{tabular}{llcr}
\hline Partei & & Senat & Abgeordnetenhaus \\
Partido Colorado & Bordaberry & 743 & 28 \\
& Battle & 5 & 12 \\
& Vasconcellos & 1 & 1 \\
Partido Colorado & insgesamt: & $13 / 14$ & 41 \\
Partido Nacional & Ferreira & 8 & 30 \\
& Aguerrondo & 4 & 10 \\
Partido Nacional & insgesamt: & 12 & 40 \\
PDS (Frente Amplio) & Seregni & 5 & 18 \\
\hline Insgesamt: & & 30 & 99
\end{tabular}

41 Vgl. "Lo que expresan las cifras definitivas“, in: Carta de Montevideo, Bd. 7, No. 346 vom 21.2 .1972 ,

42 Die Aufteilung der Mandate auf die Präsidentschaftskandidaten gibt den echten Zersplitterungsgrad der Gruppen und Grüppchen im neuen Parlament nur sehr ungenau wieder, da viele Gruppen zwar einen gemeinsamen Präsidentschaftskandidaten unterstützten, für Senat und Abgeordnetenhaus aber eigene Listen präsentiert hatten. So verteilen sich die Stimmen für Ferreira auf drei voneinander unabhängige Gruppierungen - seine eigene Gruppe erhielt nur 5 Senatoren und 18 Abgeordnete. Die Senatoren und Abgeordneten des PDC verteilen sich auf fünf selbständige Listen, und auch die unter Bordaberry aufgeführten Mandate entfielen nur zum Teil auf dessen Gruppe. Der Rest (ein Senator und drei Abgeordnete) wurde über die Liste „515* unter der Führung Glauco Segovias gewählt. Die Tabelle vermittelt so das Bild einer "relativen Konzentration ${ }^{\alpha}$, die aber in Wirklichkeit nicht gegeben ist. Angaben aus "La integración de ambas cámaras ${ }^{\alpha}$, in: Carta de Montevideo, Bd. 7, No. 246 vom 21. 2. 1972, S. 5.

43 Die Situation des Präsidenten wird geringfügig verbessert durch die Tatsache, daß der gewählte Vizepräsident gleichzeitig Präsident des Senates (und der Asamblea General) mit Sitz und Stimme ist. 
Von den Colorado-Zeitungen wurde das Ergebnis vor allem als großer Sieg der demokratischen Kräfte gefeiert ${ }^{44}$. Die Mehrheitsverhältnisse in beiden Häusern des Parlaments zwingen jedoch zu einiger Skepsis ${ }^{45}$. Die Gruppen, die den Präsidenten unterstützten, verfügen im Parlament nur über wenig mehr als 25 Prozent der Sitze. Bordaberrys erste Aufgabe mußte es deswegen sein, die übrigen Colorado-Gruppen zur Zusammenarbeit zu bewegen, wollte er nicht die von Pacheco begonnene Politik der Konfrontation zwischen Exekutive und Legislative fortsetzen, ohne einen Versuch der Kooperation gemacht zu haben. Aber auch eine solche Zusammenarbeit, die sich im Senat auf 14 (einschließlich des Vizepräsidenten) und dem Abgeordnetenhaus 41 Mandate hätte stützen können, reichte nicht aus, um mit parlamentarischer Unterstützung regieren zu können. Bordaberry bemühte sich deshalb nach Gesprächen mit den Mitbewerbern um die Präsidentschaft in den eigenen Reihen (Batlle und Vasconcellos) auch in Gesprächen mit den Spitzenkandidaten des Partido Nacional um eine Übereinkunft für die zukünftige Regierungsarbeit, während er jede Úbereinkunft mit dem Frente Amplio kategorisch zurück wies ${ }^{46}$.

Aber selbst wenn eine Übereinkunft zwischen allen Blanco- und Coloradogruppen zustande gekommen wäre, hätte es damit noch keine Garantie für eine erfolgreiche Zusammenarbeit gegeben. Übereinkünfte $z$ wischen politischen Gruppierungen in Uruguay sind bisher stets sehr kurzlebig gewesen, und in den meisten Fällen sind sie zerbrochen, noch ehe sie Resultate gezeitigt hatten ${ }^{47}$. Langfristig fällt es auch schwer, sich eine Zusammenarbeit zwischen dem konservativen Bordaberry und dem am äußersten linken Flügel der Colorados lavierenden Vasconcellos vorzustellen, und eine Allianz Bordaberry/Ferreira - zahlenmäßig die einzige Garantie für eine kontinuierliche Regierungstätigkeit auf Mehrheitsbasis - müßte am unterschiedlichen Naturell der beiden Kandidaten scheitern. Die innere Logik einer solchen Koalition hätte Ferreira auch schon bald zur Distanz zwingen müssen, da seine Chancen bei der nächsten Wahl durch allzu enge Zusammenarbeit mit der Regierung eher geschmälert worden wären. Zusätzliche Profilierung konnte Ferreira eher von entschiedener Opposition erwarten. Außerdem mußte es Ferreira schwerfallen, als mit Abstand stärkster Kandidat die untergeordnete Rolle in einer Koalition mit dem wesentlich schwächeren Kandidaten zu übernehmen. In dieser Situation erklärte sich die Mehrheit des Blanco-Directorios auf Betreiben Ferreiras nur dazu bereit, auf parlamentarischer Basis mit der Regierung an der Erstellung aus nationalen Gründen notwendiger Gesetzesvorlagen mitzuarbeiten, lehnte aber eine direkte Beteiligung an der Regierung $a b^{48}$. Von der zweiten Blanco-Gruppe unter Führung des alternden Caudillos Martín Echegoyen war zwar mehr Bereitschaft zur Kooperation zu erwarten, aber deren Mandate reichten zu einer stabilen Mehrheit nicht aus. Außerdem hätte ein solcher Alleingang die mühsam erreichte Übereinkunft zwischen

44 S. z. B. „Gran triunfo de los demócratas“, in: La Mañana vom 29. 11. 1971.

45 Darauf hatten einige argentinische Beobachter bereits vor der Wahl hingewiesen. S. z. B. „Los problemas vendrán después“ - "die Probleme werden später kommen“, in: Visión (Buenos Aires), Bd. 39, No. 23 vom 20. 11. 1971, S. $26 \mathrm{ff}$.

46 S. „Bordaberry logra primer entendimiento con el nacionalismo“, in: Carta de Montevideo, Bd. 7, No. 346 vom 21. 2. 1972, S. 3.

47 Für eine Reihe von illustrativen Beispielen vgl. Kerbusch, E. J., Das uruguayische Regierungssystem, a.a. O., S. $120 f$.

48 S. „Decisión Blanca: apoyo al nivel parlamentario“, in: Carta de Montevideo, Bd. 7, No. 347 vom 28. 2. 1972 , S. 3. 
den beiden Blanco-Gruppen, die erst im März zur Bildung einer einheitlichen Parteiführung (directorio único) geführt hatte, wieder zunichte machen müssen ${ }^{49}$.

$\mathrm{Da}$ vom Frente Amplio mit bedingungsloser Opposition gegen die neue Regierung zu rechnen ist, geht man wohl kaum ein großes Risiko ein, wenn man die Voraussage wagt, daß es auch Bordaberry langfristig nicht möglich sein wird, mit Unterstützung des Parlaments zu regieren. Eine Alternative, die noch vor wenigen Jahren beinahe ausgeschlossen erschien ${ }^{50}$, wird heute ganz offen diskutiert: Die Militärs, aufgewertet durch ihren politischen Einsatz im Rahmen des Ausnahmezustandes, könnten versuchen, den versagenden Politikern das Heft aus der Hand zu nehmen. Mit demokratischen Strukturen westlichen Musters ist es Uruguay bisher nicht gelungen, einen Modernisierungsprozeß in Gang zu bringen. Es kann nicht mehr ausgeschlossen werden, daß der „ökonomischen Lateinamerikanisierung" des früher eher europäisch wirkenden Landes die "politische Lateinamerikanisierung " - gekennzeichnet durch die Häufigkeit der Machtübernahme ohne vorherige demokratische Legitimation - folgen wird ${ }^{51}$.

49 Die konservativen Blanco-Gruppen hatten in einer Resolution deutlich gemacht, daß sie auch zu mehr und direkterer - Kooperation bereit seien als die Mehrheit des Directorio Bordaberry zuzugestehen bereit war. Vgl. "A poyo al cogobierno ${ }^{\alpha}$, in: Carta de Montevideo, Bd. 7, No. 347 vom 28. 2. 1972, S. 4.

50 Noch 1969 - also nach mehr als einem Jahr permanentem Ausnahmezustand - hatten $63 \%$ der Uruguayer bei einer Befragung erklärt, sie hielten einen Staatsstreich oder eine Diktatur für unmöglich. S. Gallup Uruguay, Informe Gallup 1969, Montevideo 1969, S. 15.

51 Zum Begriff s. Nohlen, D., Politischer Wandel durch Wahlen: Der Fall Uruguay, a.a.O., S. 4. 


\section{The Elections of November 28, 1971 in Uruguay}

\section{By ERNST-J. Kerbusch}

The present situation in Uruguay is characterized by economic stagnation and the loss of consensus of a traditional political system. On the basis of the history and results of the November 1971 elections and the ensuing formation of the government, this paper tries to explore critical historical aspects of the institutional development of this country whose democracy once was considered a model for Latin America.

For more than a century only two political parties were realistic candidates for the formation of the government and they even anchored this system in the Constitution: The Partido Colorado (progressive, rooted in urban centers) and the Partido Blanco (conservative, strong in the countryside). Different factions, however, always emerge within the parties. They put up their own candidates and agree upon any one of them only after the election results are in. In Parliament they form coalitions which confront the Executive with an absolute majority. In 1971 for the first time a third party, the Frente Amplio, made up of the forces of the democratic left, participated in the elections. Election time was marked not only by this new development but also by the activities of the urban guerilla (Tupamaros) and the serious economic situation. The Colorado Party won a very narrow victory of $0,7 \%$ over the Blancos. The Frente Amplio finished an unexpected poor third with $18,3 \%$ of the votes.

The immobility of the traditional voting potential is being transferred to the process of government formation and translated into patt situations between Executive and Parliament and a low potential for dynamic development. Thus, democratic structures of the western type appear unable to initiate a process of modernization. An alternative, quite unheard of only a few years ago, is now being seriously discussed: The military whose prestige has been enhanced by their political engagement during frequent applications of martial law, could be tempted to take over from the faltering politicians.

\section{The Political Rights of Women in Argentinia, a Historical Outline}

\section{By Juan Jose Reyven}

In 1862 for the first time a woman took part in a communal election held in San Juan, Argentinia. But not until 1914 did the women achieve the franchise for provincial elections. Since then a tremendous amount of resolutions and bills on the political rights of women had passed parliament without success. There was an extensive discussion on which kind of qualifications should be insisted upon as a prerequisite for the franchise. Another question was, whether or not the franchise should be combined with the duty of military service. These various unsuccessful attempts to pass a piece of legislation which everybody thought to be necessary, show which kind of difficulties arise when new political structures are introduced into traditional society. The text of the 1947 Act on the political rights of women, with recent amendments, is also added.

\section{Divorce in Ghana}

\section{By KwAME OPOKU}

Until the passing of the Matrimonial Causes Act of 1971, English statute and case law governed the dissolution of Ordinance (English law) marriages in Ghana by virtue of the High Court Ordinance which stated that the jurisdiction of the High 